\title{
Beta-2 Microglobulin in Whole Unstimulated Saliva Can Effectively Distinguish Between Sjögren's Syndrome and Non-Autoimmune Sicca Symptoms
}

\author{
Janett RIEGA-TORRES, ${ }^{1}$ Guillermo DELGADO-GARCÍA, ${ }^{2}$ Julio César SALAS-ALANÍS, ${ }^{3}$ \\ Cassandra SKINNER-TAYLOR, ${ }^{1}$ Lorena PÉREZ-BARBOSA, ${ }^{1}$ Mario GARZA-ELIZONDO, ${ }^{1}$ \\ Celia Nohemí SÁNCHEZ-DOMÍNGUEZ, ${ }^{4}$ Luis Ángel CECEÑAS-FALCÓN, ${ }^{5}$ \\ Karim MOHAMED-NORIEGA, ${ }^{6}$ Jesús MOHAMED-HAMSHO, ${ }^{6}$ David VEGA-MORALES ${ }^{1}$ \\ ${ }^{1}$ Division of Rheumatology, University Hospital, Autonomous University of Nuevo León, Monterrey, Mexico \\ ${ }^{2}$ Department of Internal Medicine, University Hospital, Autonomous University of Nuevo León, Monterrey, Mexico \\ ${ }^{3}$ Department of Basic Sciences, University of Monterrey, Monterrey, Mexico \\ ${ }^{4}$ Department of Biochemistry And Molecular Medicine, Faculty of Medicine, Autonomous University of Nuevo León, Monterrey, Mexico \\ ${ }^{5}$ Division of Anatomic Pathology, University Hospital, Autonomous University of Nuevo León, Monterrey, Mexico \\ ${ }^{6}$ Department of Ophthalmology, University Hospital, Autonomous University of Nuevo León, Monterrey, Mexico
}

\begin{abstract}
Objectives: This study aims to describe salivary beta-2 microglobulin (sB2M) levels in our setting and to assess the performance of sB2M for the diagnosis of Sjögren's syndrome (SS).

Patients and methods: This cross-sectional, comparative study included 192 SS patients (2 males, 190 females; mean age 53.1 years; range 23 to 84 years) and 64 healthy controls ( 1 male, 63 females; mean age 46.9 years; range 21 to 82 years). Patients were divided into three groups as those with primary SS, secondary SS, and sicca non-Sjögren's syndrome (snSS). sB2M was measured by enzyme-linked immunosorbent assay in whole unstimulated saliva $(\mathrm{ng} / \mathrm{mL}$ ). Differences in $\mathrm{SB} 2 \mathrm{M}$ were evaluated using the Kruskal-Wallis test. Receiver operating curves were generated to determine the performance of sB2M for distinguishing between SS and non-autoimmune snSS groups, and between SS group and healthy controls. Results: The primary SS and secondary SS groups had a significantly higher concentration of sB2M than the other two groups. There was no significant difference in the concentration of SB2M between primary SS and secondary SS groups, and neither between snSS group and healthy controls. The receiver operating curve analysis for distinguishing SS and snSS showed an area under the curve of 0.661 ( $95 \%$ confidence interval $0.590-0.728, p=0.0001$ ) with an optimal cutoff value of $0.582 \mathrm{ng} / \mathrm{mL}$. Sensitivity, specificity, positive predictive value, and negative predictive value were $68.7 \%, 59.3 \%, 20.2 \%$, and $92.7 \%$, respectively. The reported prevalence of SS in Mexico was considered when calculating the last two values.

Conclusion: In our setting, sB2M effectively distinguished between SS patients and non-autoimmune sicca symptoms. Including sB2M in our conventional diagnostic arsenal may assist in the evaluation of patients in whom SS is suspected; however, further studies are needed to clarify this hypothesis.

Keywords: Beta-2 microglobulin; diagnosis; saliva; Sjögren's syndrome.
\end{abstract}

Elevated salivary beta-2 microglobulin (sB2M) has been already reported in both primary Sjögren's syndrome (pSS) and secondary SS (sSS). ${ }^{1,2} \mathrm{~B} 2 \mathrm{M}$ is present on the plasmalemma of many cells, including infiltrating lymphocytes and salivary gland epithelial cells, and its increased levels in saliva may be related to salivary gland inflammatory activity in SS. ${ }^{3}$ Sialochemistry

Received: November 11, 2016 Accepted: February 20, 2017 Published online: May 25, 2017

Correspondence: Guillermo Delgado-García, MD. Department of Internal Medicine, University Hospital, Autonomous University of Nuevo León, 64460 Monterrey, Mexico. e-mail: grdelgadog@gmail.com

○2017 Turkish League Against Rheumatism. All rights reserved. 
has been proposed as a useful tool in SS. ${ }^{2,4}$ Asashima et al. ${ }^{5}$ recently reported cutoff levels of sB2M for differentiating SS patients from other patients in a Japanese sample. To the best of our knowledge, sB2M levels are currently unknown in our population. Therefore, in this study, we aimed to describe sB2M levels in our setting and to assess the performance of $\mathrm{sB2M}$ for the diagnosis of SS.

\section{PATIENTS AND METHODS}

This cross-sectional, comparative study included 192 SS patients (2 males, 190 females; mean age 53.1 years; range 23 to 84 years) recruited from the outpatient clinic between July 2012 and July 2014 and 64 healthy controls (HC; 1 male, 63 females; mean age 46.9 years; range 21 to 82 years). Patients were divided into three groups as those with pSS, sSS, and sicca non-Sjögren's syndrome (snSS) (each group $n=64$ ). HC were recruited from among staff and patients' companions who were non-relatives. Of the SS patients, those with history of human immunodeficiency virus, hepatitis B and C, head and neck radiation therapy, sarcoidosis, amyloidosis, immunoglobulin G4-related disease, chronic kidney disease, lymphoma, or multiple myeloma were excluded. All conditions mentioned in this study were diagnosed according to their corresponding criteria. ${ }^{6-9}$ snSS was defined on clinical and laboratory grounds. None of these patients had a history of autoimmune disease. The study protocol was approved by the institutional review board. A written informed consent was obtained from all individuals. The study was conducted in accordance with the principles of the Declaration of Helsinki.

Beta-2 microglobulin was determined by an enzyme-linked immunosorbent assay (ab108885, Abcam, Cambridge, UK) and results were expressed as nanograms per milliliter $(\mathrm{ng} / \mathrm{mL})$. As noted in a previous report, ${ }^{10}$ our protocol was similar to that formerly described by Castro et al. ${ }^{4}$ Unstimulated whole saliva samples were collected at the fasting state, in the morning, by passive spitting into containers over 15 minutes. These samples were thus centrifuged at $14,000 \times \mathrm{g}$ for 20 minutes at $+4^{\circ} \mathrm{C}$ prior to assaying.

\section{Statistical analysis}

The Kolmogorov-Smirnov test was used to evaluate whether the distribution of continuous variables was normal. Discrete variables were evaluated using exact $\mathrm{r} \times \mathrm{c}$ contingency table analysis or Chi-squared test. Continuous variables were evaluated using one-way analysis of variance or Kruskal-Wallis test. If the oneway analysis of variance was positive, then the Student-Newman-Keuls test was performed for pairwise comparison of groups. If the Kruskal-Wallis test was positive, then a test for pairwise comparison of groups was performed according to Conover. ${ }^{11}$ Receiver operating characteristic (ROC) curves were generated to determine the performance of $\mathrm{sB} 2 \mathrm{M}$ for distinguishing SS and snSS/HC. Sensitivity, specificity, positive predictive value (PPV), and negative predictive value (NPV) were thus estimated. The reported prevalence of SS in Mexico was considered when calculating the last two values. ${ }^{12}$ Discrete variables are expressed as frequency counts and percentages. Continuous variables are expressed as mean \pm standard deviation or median (P25, P75). Except for exact contingency table analysis, statistical analyses were performed using MedCalc for Windows, version 13.3 (MedCalc Software, Ostend, Belgium). Exact contingency table analysis was performed using Statistics to Use. ${ }^{13}$ A $p$ value of $<0.05$ was considered statistically significant. MedCalc was also used to generate the ROC curves. The boxplot was made using GraphPad Prism for Windows, version 5.01 (GraphPad Software, San Diego, USA).

\section{RESULTS}

Healthy controls were younger than the other groups $(p<0.05)$. Although there was no statistically significant difference in the distribution of sex between groups, the distribution of type 2 diabetes mellitus, arterial hypertension, and fibromyalgia was different between groups $(p<0.015)$. There was no statistically significant difference in the proportion of users of steroids, xerogenic medication, and hydroxychloroquine between pSS and sSS groups. However, the use of methotrexate was more common in those with sSS $(p=0.009)$ (Table 1). 


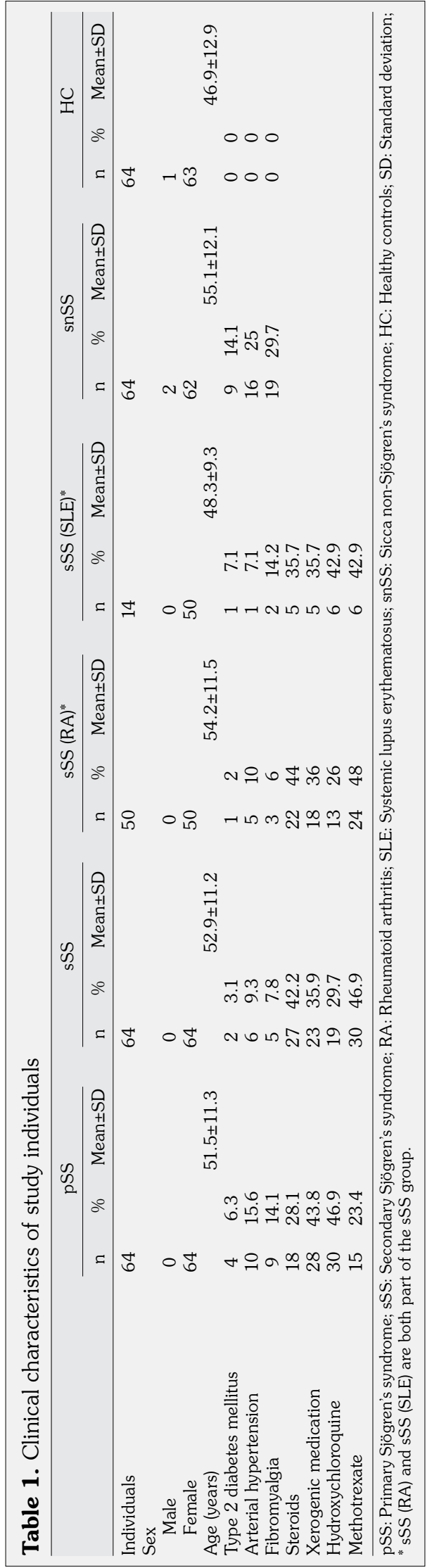

There was a statistically significant difference in sB2M levels between groups $(p<0.000001)$. The pSS and sSS groups had a comparatively higher concentration of sB2M than the other two groups $(p<0.005)$. There was no statistically significant difference in the concentration of sB2M between pSS and sSS groups, and neither between snSS group and HC (Table 2 and Figure 1a). The ROC curve analysis for distinguishing $\mathrm{SS}$ and snSS based on sB2M showed an area under the curve of 0.661 (95\% confidence interval [CI]: 0.590-0.728, $\mathrm{p}=0.0001$ ) with an optimal cutoff value of $0.582 \mathrm{ng} / \mathrm{mL}$ (Figure 1b). Sensitivity, specificity, PPV, and NPV were 68.7\% (95\% CI: 60-76.6\%), 59.3\% (95\% CI: 46.4-71.5\%), 20.2\% (95\% CI: $12.3-30.3 \%)$, and $92.7 \%$ (95\% CI: 86-96.8\%), respectively. The area under the curve for distinguishing SS and $\mathrm{HC}$ was 0.747 (95\% CI: 0.679-0.807, p<0.0001). At a cutoff level of $0.625 \mathrm{ng} / \mathrm{mL}$, sensitivity, specificity, PPV, and NPV were 66.4\% (95\% CI: 57.5-74.5\%), 76.5\% (95\% CI: 64.3-86.2\%), 29.7\% (95\% CI: 18.2-43.5\%), and 93.8\% (95\% CI: 88.4-97.2\%), respectively (Figure 1c).

\section{DISCUSSION}

In this study, we found that $\mathrm{B} 2 \mathrm{M}$ in whole unstimulated saliva can distinguish between SS and snSS, and also between SS and HC. In Mexico, a sB2M level below the cutoff can rule out this diagnosis (NPV >92\%). PPV and NPV can be recalculated for different populations using some formulas based on Bayes' theorem (available in MedCalc). We also showed that sB2M can particularly distinguish pSS patients from those with sicca symptoms but no autoimmune disease.

Maddali Bongi et al. ${ }^{14}$ studied three decades ago the diagnostic performance of $\mathrm{sB2M}$ in a small sample. In this study, the diagnosis of pSS was established based on the criteria proposed by Fox et al., ${ }^{15}$ which are no longer in use. ${ }^{14}$ Asashima et al. ${ }^{5}$ recently reported cutoff levels of sB2M for differentiating SS patients from other patients and $\mathrm{HC}$ in a Japanese sample. While on a first level our results were similar to theirs (i.e., sB2M was higher in pSS group compared to $\mathrm{HC}$, and no difference between pSS and sSS groups), there were at least two relevant discrepancies between 
Table 2. Salivary beta-2 microglobulin $(\mathrm{ng} / \mathrm{mL})$ in whole unstimulated saliva

\begin{tabular}{lcccccc}
\hline & pSS & sSS & sSS (RA)* & sSS (SLE)* & snSS & HC \\
\hline Individuals & 64 & 64 & 50 & 14 & 64 & 64 \\
Median & 0.7109 & 0.76970 & 0.84450 & 0.63130 & 0.52780 & 0.35950 \\
P25 & 0.4420 & 0.52620 & 0.62500 & 0.22890 & 0.27330 & 0.27190 \\
P75 & 1.1756 & 1.29060 & 1.39090 & 0.88850 & 0.81530 & 0.62500 \\
\hline $\begin{array}{l}\text { pSS: Primary Sjögren's syndrome; sSS: Secondary Sjögren's syndrome; RA: Rheumatoid arthritis; SLE: Systemic lupus erythematosus; snSS: Sicca } \\
\text { non-Sjögren's syndrome; HC: Healthy controls; P25: 25 }\end{array}$ \\
\hline
\end{tabular}

them: (i) the magnitude of sB2M levels, and (ii) the results of the $\mathrm{ROC}$ curve.

Addressing the first point, they reported a mean level of $\mathrm{sB} 2 \mathrm{M}$ of $5.3 \pm 4.6 \mathrm{mg} / \mathrm{L}$ (or $5,300 \pm 4,600 \mathrm{ng} / \mathrm{mL}$ ) in those with $\mathrm{pSS}^{5}$ In our study, the highest concentration of $\mathrm{sB} 2 \mathrm{M}$ was found in a pSS patient, but it was $9.1 \mathrm{ng} / \mathrm{mL}$ (or $0.0091 \mathrm{mg} / \mathrm{L}$ ) (Figure 1a). As a matter of fact, we reported a median level of sB2M of 0.71 (0.442, 1.175) ng/mL (or 0.00071 [0.000442, $0.001175] \mathrm{mg} / \mathrm{L}$ ) in those with pSS (Table 2). Although this disparity could be attributed to variations in salivary flow, ${ }^{16}$ it is not confined only to this group (i.e., pSS), and $\mathrm{HC}$ have no reason
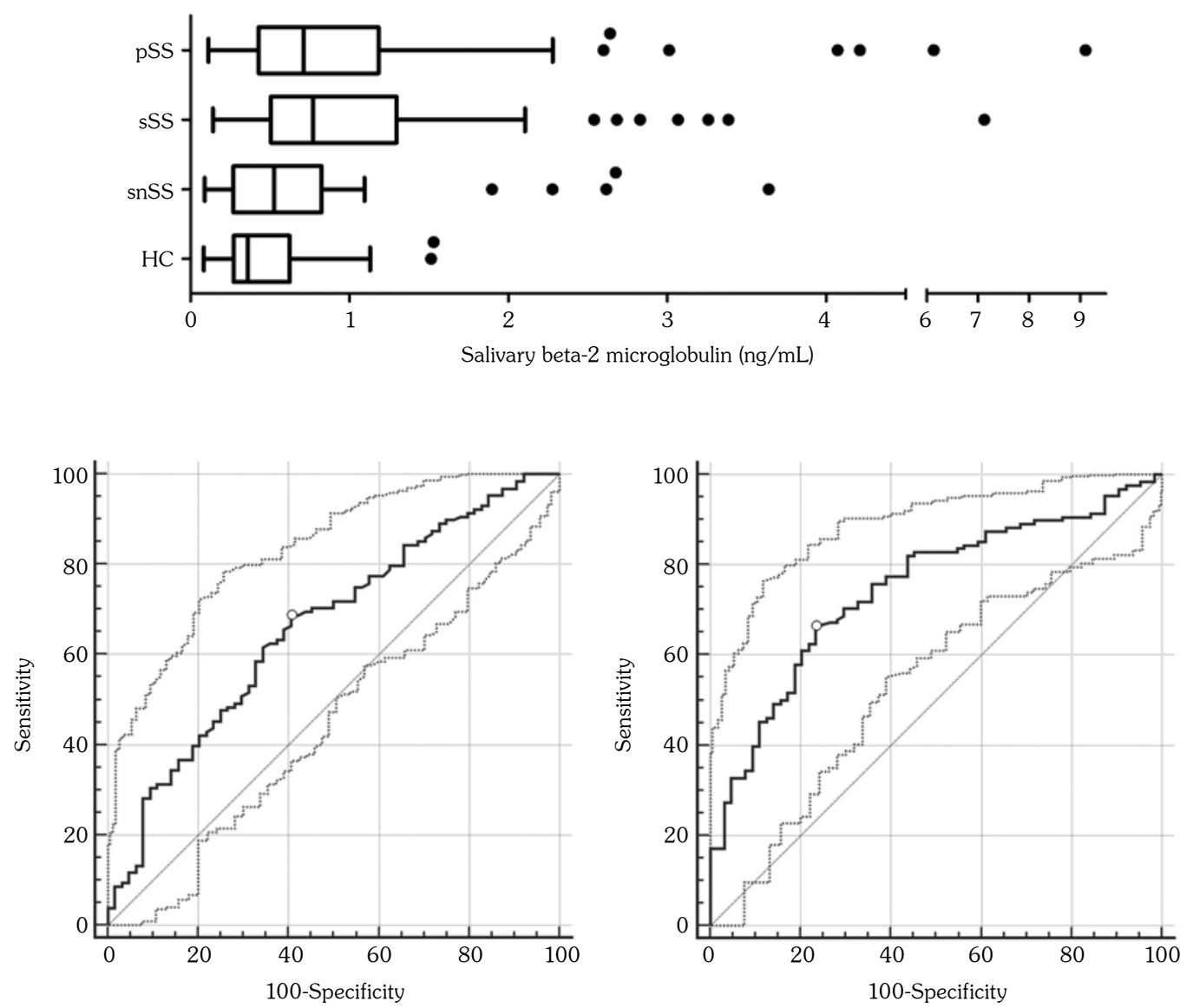

Figure 1. (a) Salivary beta-2 microglobulin in whole unstimulated saliva. (b) Performance characteristics of salivary beta-2 microglobulin for distinguishing Sjögren's syndrome and sicca non-Sjögren's syndrome, and (c) Sjögren's syndrome and healthy controls. 95\% confidence bounds for the receiver operating characteristic curves are also displayed. 
to have salivary flow alterations: Asashima et al. ${ }^{5}$ reported a mean level of sB2M of $1.2 \pm 0.7 \mathrm{mg} / \mathrm{L}$ (or $1,200 \pm 700 \mathrm{ng} / \mathrm{mL}$ ) in $\mathrm{HC}$, while we reported a median level of $0.3595(0.2719,0.625) \mathrm{ng} / \mathrm{mL}$ (or 0.0003595 [0.0002719, 0.000625$] \mathrm{mg} / \mathrm{L}$ ) in the same group (Table 2). It is worth noting that, in measuring sB2M, different products were employed: they used a latex agglutination photometric immunoassay (Eiken Chemicals Co., Tokyo, Japan), and we used an in vitro enzyme-linked immunosorbent assay (ab108885, Abcam, Cambridge, UK), which was designed for the quantitative measurement of B2M levels in saliva and other fluids. We do not know if this latex agglutination photometric immunoassay was designed, or has been previously used, in saliva. Its pamphlet is not available in English (retrieved on September 29, 2016: http://www. eiken.co.jp/products_technique/pamph/9009. pdf). Asashima et al. ${ }^{17}$ previously reported the highest level of sB2M in the literature. In this case report, they used the same measurement method (i.e., latex agglutination photometric immunoassay).

Addressing the second point, on ROC curve analysis, Asashima et al. ${ }^{5}$ reported a sB2M cutoff level of $2.3 \mathrm{mg} / \mathrm{L}(2,300 \mathrm{ng} / \mathrm{mL})$ to differentiate the SS group from the non-SS group. None of the individuals evaluated by us (including both patients and $\mathrm{HC}$ ) reached this cutoff level. Due to the above, we did not recalculate PPV and NPV using their results.

$\mathrm{Hu}$ et al. ${ }^{18}$ previously proposed to study sB2M in order to distinguish patients with pSS from those with sicca symptoms but no autoimmune disease. Our snSS group represents such patients. Baldini et al. ${ }^{19}$ also included a snSS group in their study. Specifically addressing this point, the ROC curve analysis showed an area under the curve of 0.648 (95\% CI: 0.559-0.730, $\mathrm{p}=0.0023$ ) with an optimal cutoff value of $0.638 \mathrm{ng} / \mathrm{mL}$ (not shown in Figure 1). Sensitivity, specificity, PPV, and NPV were 64\% (95\% CI: 51.1-75.7\%), 60.94\% (95\% CI: 47.972.9\%), $4.8 \%$ (95\% CI: $0.8-14.8 \%$ ), and $98.2 \%$ (95\% CI: 92.2-99.9\%), respectively. In summary, in those with sicca symptoms and no history of autoimmune disease, a sB2M level below this cutoff can rule out the diagnosis of pSS (NPV $>98 \%$ ) in our setting.
Although our study was not underpowered, we did not find a statistically significant difference in sB2M between pSS and sSS groups. This is in contrast to the results from Baldini et al. ${ }^{19}$ but similar to that reported by Asashima et al. ${ }^{5}$ Unlike other investigators, ${ }^{5,18}$ we only included patients with sicca symptoms, since the idea behind our study was to develop an additional tool to benefit in the diagnosis of those in whom SS is suspected (i.e., in those with sicca symptoms). In our study, fibromyalgia was more common in those with sicca symptoms but no autoimmune disease (snSS group). However, proteomic analysis of saliva has been already performed in patients with fibromyalgia and sB2M was not reported to be differentially expressed. ${ }^{20}$ Therefore, this difference in the proportion of patients with fibromyalgia probably did not influence our results. Previous studies with $\mathrm{sB} 2 \mathrm{M}$ have used parotid saliva. ${ }^{2,3,21,22}$ Whole unstimulated saliva reflects the total contribution from all the salivary glands. ${ }^{16}$ As Asashima et al., ${ }^{5}$ we also employed unstimulated whole saliva, because this collection method is rapid, simple, inexpensive, and non-invasive, and it is thus more likely to be used in real-life clinical settings.

Our study has some limitations. For example, there were some baseline differences between groups. HC were comparatively younger than the rest of the groups. However, sB2M was not correlated with age in a previous study. ${ }^{22}$ The frequency of type 2 diabetes mellitus and arterial hypertension was also different between groups. However, as far as we know (and at least in PubMed/MEDLINE), there are no reports about the effect of diabetes mellitus (either type 1 or 2 ) or arterial hypertension on $\mathrm{sB2M}$. Therefore, the possible influence of these two conditions on $\mathrm{sB} 2 \mathrm{M}$ is currently unknown.

In conclusion, while sB2M may not be the ideal screening method for $\mathrm{SS}$, it may be used as an adjunctive diagnostic test ${ }^{22}$ and, in our setting, it can effectively distinguish between SS patients (either pSS or sSS) and those with sicca symptoms but no autoimmune disease. Including sB2M in our conventional diagnostic arsenal may assist in the evaluation of patients in whom SS is suspected; however, further studies are needed to clarify this hypothesis. 


\section{Declaration of conflicting interests}

The authors declared no conflicts of interest with respect to the authorship and/or publication of this article.

\section{Funding}

The authors received no financial support for the research and/or authorship of this article.

\section{REFERENCES}

1. Talal N, Grey HM, Zvaifler N, Michalski JP, Daniels TE. Elevated salivary and synovial fluid beta2microglobulin in Sjogren's syndrome and rheumatoid arthritis. Science 1975;187:1196-8.

2. Baldini C, Gallo A, Perez P, Mosca M, Alevizos I, Bombardieri S. Saliva as an ideal milieu for emerging diagnostic approaches in primary Sjögren's syndrome. Clin Exp Rheumatol 2012;30:785-90.

3. Ryu OH, Atkinson JC, Hoehn GT, Illei GG, Hart TC. Identification of parotid salivary biomarkers in Sjogren's syndrome by surface-enhanced laser desorption/ionization time-of-flight mass spectrometry and two-dimensional difference gel electrophoresis. Rheumatology (Oxford) 2006;45:1077-86.

4. Castro J, Jiménez-Alonso J, Sabio JM, Rivera-Cívico F, Martín-Armada M, Rodríguez MA, et al. Salivary and serum beta2-microglobulin and gamma-glutamyltransferase in patients with primary Sjögren syndrome and Sjögren syndrome secondary to systemic lupus erythematosus. Clin Chim Acta 2003;334:225-31.

5. Asashima $\mathrm{H}$, Inokuma $\mathrm{S}$, Onoda M, Oritsu M. Cutoff levels of salivary beta2-microglobulin and sodium differentiating patients with Sjögren's syndrome from those without it and healthy controls. Clin Exp Rheumatol 2013;31:699-703.

6. Goules AV, Tzioufas AG, Moutsopoulos HM. Classification criteria of Sjögren's syndrome. J Autoimmun 2014;48-49:42-5.

7. Kourilovitch M, Galarza-Maldonado C, Ortiz-Prado E. Diagnosis and classification of rheumatoid arthritis. J Autoimmun 2014;48-49:26-30.

8. Yu C, Gershwin ME, Chang C. Diagnostic criteria for systemic lupus erythematosus: a critical review. J Autoimmun 2014;48-49:10-3.

9. Häuser W, Wolfe F. Diagnosis and diagnostic tests for fibromyalgia (syndrome). Reumatismo 2012;64:194-205.

10. Garza-García F, Delgado-García G, Garza-Elizondo M, Ceceñas-Falcón LÁ, Galarza-Delgado D, Riega-Torres
J. Salivary $\beta 2$-microglobulin positively correlates with ESSPRI in patients with primary Sjögren's syndrome. Rev Bras Reumatol Engl Ed 2017;57:182-184.

11. Conover W. Practical Nonparametric Statistics. 3rd ed. New York: John Wiley \& Sons; 1999.

12. Sánchez-Guerrero J, Pérez-Dosal MR, CárdenasVelázquez F, Pérez-Reguera A, Celis-Aguilar E, SotoRojas AE, et al. Prevalence of Sjögren's syndrome in ambulatory patients according to the AmericanEuropean Consensus Group criteria. Rheumatology (Oxford) 2005;44:235-40.

13. Kirkman T. Statistics to Use [Internet]. 1996 Available from: http://www.physics.csbsju.edu/stats/ [Access: May 30, 2016].

14. Maddali Bongi S, Campana G, D’Agata A, Palermo C, Bianucci $G$. The diagnosis value of beta 2 -microglobulin and immunoglobulins in primary Sjögren's syndrome. Clin Rheumatol 1995;14:151-6.

15. Fox RI, Robinson CA, Curd JG, Kozin F, Howell FV. Sjögren's syndrome. Proposed criteria for classification. Arthritis Rheum 1986;29:577-85.

16. van der Geest SA, Markusse HM, Swaak AJ. Beta 2 microglobulin measurements in saliva of patients with primary Sjögren's syndrome: influence of flow. Ann Rheum Dis 1993;52:461-3.

17. Asashima $\mathrm{H}$, Inokuma $\mathrm{S}$, Nakachi $\mathrm{S}$, Matsuo $\mathrm{Y}$, Rokutanda R, Hagiwara K, et al. Extremely high salivary $\beta(2)$-microglobulin and $\mathrm{Na}(+)$ levels in a Sjögren syndrome patient. Int $J$ Rheum Dis 2012;15:31-3.

18. Hu S, Gao K, Pollard R, Arellano-Garcia M, Zhou $\mathrm{H}$, Zhang $\mathrm{L}$, et al. Preclinical validation of salivary biomarkers for primary Sjögren's syndrome. Arthritis Care Res (Hoboken) 2010;62:1633-8.

19. Baldini C, Giusti L, Ciregia F, Da Valle Y, Giacomelli C, Donadio E, et al. Proteomic analysis of saliva: a unique tool to distinguish primary Sjögren's syndrome from secondary Sjögren's syndrome and other sicca syndromes. Arthritis Res Ther 2011;13:194.

20. Bazzichi L, Ciregia F, Giusti L, Baldini C, Giannaccini G, Giacomelli C, et al. Detection of potential markers of primary fibromyalgia syndrome in human saliva. Proteomics Clin Appl 2009;3:1296-304.

21. Swaak AJ, Visch LL, Zonneveld A. Diagnostic significance of salivary levels of beta 2-microglobulin in Sjögren's syndrome. Clin Rheumatol 1988;7:2834.

22. Markusse HM, Otten HG, Vroom TM, Smeets TJ, Fokkens N, Breedveld FC. The diagnostic value of salivary fluid levels of beta 2-microglobulin, lysozyme and lactoferrin for primary Sjögren's syndrome. Clin Rheumatol 1992;11:521-5. 